Relations industrielles

Industrial Relations

\title{
Jean MEHLING : Analyse socio-économique d'une grève. Montréal, HEC et Beauchemin, 1963, 218 pp.
}

\section{Jacques Gagnon}

Volume 41, numéro 3, 1986

URI : https://id.erudit.org/iderudit/050250ar

DOI : https://doi.org/10.7202/050250ar

Aller au sommaire du numéro

Éditeur(s)

Département des relations industrielles de l'Université Laval

ISSN

0034-379X (imprimé)

1703-8138 (numérique)

Découvrir la revue

Citer ce compte rendu

Gagnon, J. (1986). Compte rendu de [Jean MEHLING : Analyse socio-économique d'une grève. Montréal, HEC et Beauchemin, 1963, 218 pp.] Relations industrielles / Industrial Relations, 41(3), 670-671. https://doi.org/10.7202/050250ar

Tous droits réservés (C Département des relations industrielles de l'Université Laval, 1986
Ce document est protégé par la loi sur le droit d'auteur. L'utilisation des services d'Érudit (y compris la reproduction) est assujettie à sa politique d'utilisation que vous pouvez consulter en ligne.

https://apropos.erudit.org/fr/usagers/politique-dutilisation/ 
ans l'augmentation des salaires et congés payés, et le congédiement des briseurs de grève. Par contre, ils n'ont jamais pu obtenir leurs revendications de départ. Il n'y a pas eu de cotisation syndicale obligatoire retenue à la source. Ni de consultation sur les promotions, transferts et congédiements. Ni le droit de refuser ou d'accepter individuellement les cadences de production. Le mot de la fin reste à Hélène David quị «propose que le conflit de l'amiante soit vu comme une situation où l'éclatement ouvert de la lutte de classes a engendré un conflit entre l'Église, défendant un modèle communautaire, et l'État, assurant l'équilibre de la société industrielle capitaliste. Ce modèle d'analyse permet, à partir des positions des acteurs - les ouvriers, les entreprises, l'État et l'Église - d'éclairer les contradictions dans leurs rapports réciproques et ainsi de rendre compte d'éléments non intégrés par les interprétations jusqu'ici proposées». («La grève et le Bon Dieu», in Sociologie et Sociétés, vol. 1, no 2, PUM, 1969.)

Analyse socio-économique d'une grève, par Jean Mehling, Montréal, HEC et Beauchemin, $1963,218 \mathrm{pp}$.

Comme son titre l'indique, le professeur Mehling veut lui aussi étudier un conflit de travail dans son contexte socio-économique le plus large. Mis à part les textes d'introduction et de conclusion, ses cinq chapitres portent respectivement sur la grève dans son contexte historique et conjoncturel; le rôle de la compagnie dans le développement des activités locales; sa capacité de résistance face à la grève; celle des travailleurs; et enfin, le coût de la grève et la capacité de résistance à long terme des parties en conflit. Notons que le traitement est plus équilibré que dans le volume précédent, puisque chaque chapitre compte de 24 à 50 pages ${ }^{1}$.

Véritable travail de bénédictin, cette étude ne cache ni ses lacunes, ni ses difficultés. Quatre ans après le conflit, l'auteur a épluché les dossiers des ministères fédéraux du Travail et des Mines. Il a aussi interrogé «des ouvriers réputés pour leur antipathie à l'égard du syndicat, des dirigeants syndicaux, des syndiqués réunis en groupe dans le local syndical, des religieux qui avaient pris part de diverses façons au conflit, des journalistes locaux, des hôteliers, des commerçants, des fonctionnaires, des hommes politiques, des employés de la Compagnie, etc.» (p. 51). Il a enfin mis à contribution les meilleures ressources comptables de l'École des Hautes Études commerciales. Malgré tout cela, il ne peut que conclure que «dans l'exemple de la grève de la Noranda Mines, le syndicat n'était pas en mesure, même au prix de longues recherches socio-économiques, de prendre une décision parfaitement rationnelle» (p. 214). L'auteur en prend pour preuve sa propre analyse du conflit qu'il juge trop incomplète.

Cependant, tout n'est pas négatif dans l'étude du professeur Mehling, tant s'en faut. L'auteur réussit à nous brosser le portrait fidèle d'une communauté isolée où une compagnie très puissante fait face à une force ouvrière organisée mais fragile. En effet, les travailleurs de la Noranda n'ont ni épargne ni biens de fonds pour soutenir leur grève, et ils doivent compter exclusivement sur les secours de leur centrale syndicale. Le principal enjeu du conflit est moins la hausse salariale que la "clause de retenues syndicales" et bien sûr, la capacité future de négociation des deux parties. Le conflit se termine par une victoire non équivoque de la partie patronale, après la cessation des paiements de secours de la centrale syndicale américaine. Contrairement au cas d'Asbestos, il y a eu peu d'intervention extérieure, que ce soit de l'Église, de l'État ou de l'opinion publique.

1 Il est à noter que le volume de Trudeau et al. fait allusion au conflit de RouynNoranda: «À cinq ans de distance, les mineurs de l'Abitibi étaient animés du même esprit qui avait animé ceux d'Asbestos et de Thetford-Mines. Ils retinrent même, pour la durée de leur grève, les services de M. René Rocque, ancien organisateur de la C.T.C.C., dont le nom est indissolublement lié aux événements de 1949» (Réginald Boisvert, in Trudeau, op. cit., p. 365). 
Néanmoins, la lecture de cette étude n'est pas toujours facile, malgré la clarté de ses conclusions. Les notes au bas des pages (69 en 158 pages) brisent le rythme de lecture. Certaines disgressions théoriques sont quelque peu indigestes. Enfin, quelques concepts utilisés ont mal vieilli, en particulier ceux «d'atomicité» et de «molécularité» des forces en présence. Mais on ne peut que rester impressionné par le volume de travail, la multiplicité des données, la rigueur logique, la franchise et l'honnêteté du professeur Mehling.

La grève de la United Aircraft, par Michel Pratt, Montréal, Les Presses de l'Université du Québec, 1980, 115 pp.

Parce que l'auteur affirme dès le départ son «préjugé défavorable face à la partie patronale», il est assez ironique de constater que son patronyme est le même que celui de l'exUnited Aircraft (Pratt and Whitney). Outre l'introduction et la conclusion, la chronologie et les appendices, son étude comporte quatre chapitres d'inégale longueur: les caractéristiques sociologiques des acteurs (16 pages); le rapport de forces ( 7 pages); la négociation et l'évolution du conflit ( 28 pages); la stratégie des parties ( 24 pages). Mais il faut bien convenir que les deux premiers chapitres sont meilleurs que les deux derniers.

Dans une approche résolument marxiste, ils situent la compagnie américaine et sa filiale canadienne, la centrale syndicale américaine et sa filiale locale, ainsi que le rôle de l'élite d'État canadienne et québécoise. On aurait pu facilement regrouper ces deux chapitres en un seul pour assurer un meilleur équilibre à l'ouvrage. Les troisième et quatrième chapitres sont plus discutables, surtout si on accepte la grille d'analyse marxiste. En effet, on constate que «la masse» fut loin de suivre le mouvement de son «avant-garde» syndicale, bien au contraire. Le nombre de grévistes votants passa de plus de 1500 en décembre 73 et février 74 à moins de 900 à partir de décembre 74. De plus, l'auteur s'étend vraiment trop sur le détail des propositions monétaires successivement soumises et rejetées. Malgré cela, et contrairement à Sauvé et à Mehling, il ne réussit même pas à chiffrer de façon satisfaisante les coûts de la grève pour les syndicats et les syndiqués. Ses données sont trop fragmentaires en ce qui concerne le nombre de grévistes pour chacun des 19 mois de grève (p. 75), la contribution financière totale de la centrale américaine (p. 70) et les autres contributions de grève (dont une soirée de dégustation de cidre et fromage, ainsi que la représentation d'une pièce de théâtre à la Place des Arts)...

Ce qu'on peut retenir du conflit, après lecture et relecture du texte, c'est qu'il fut beaucoup plus long, mais aussi beaucoup plus mouvementé que les deux autres que nous avons revus. Alors que les retours au travail en cours de grève se comptent par quelques centaines à Asbestos - Thetford et à Rouyn-Noranda, ils se chiffrent par plus d'un millier à Longueuil. La violence est endémique pendant une bonne partie de la grève alors qu'elle est sporadique à Asbestos et inexistante à Rouyn-Noranda. Les supports extérieurs sont venus presque exclusivement de la F.T.Q. à laquelle était affilié le syndicat local. Enfin, l'État provincial a agi de façon décisive, par l'intervention personnelle du premier ministre Bourassa. Tout cela suffit-il à faire du conflit de Longueuil «une étape aussi importante dans l'histoire sociale du Québec que la grève de l'amiante de 1949" (p. 2)? Ça reste discutable, dans la mesure où nous avons aussi questionné l'importance du conflit d'Asbestos-Thetford. Une chose est certaine, en tout cas: ni à Asbestos-Thetford, ni à Rouyn-Noranda, ni à Longueuil, la clause de retenue syndicale, jugée essentielle par le syndicat, n'a été inscrite dans le règlement final.

\section{CONCLUSION}

Pour paraphraser le petit catéchisme, nous pouvons dire que nous nous trouvons, au terme de notre recherche, devant trois styles d'étude: une analyse triomphante, celle de Trudeau, une analyse souffrante, celle de Mehling et une analyse militante, celle de Pratt. Bien 